\title{
Linx
}

Revue des linguistes de l'université Paris X Nanterre

$10 \mid 1998$

L'indicible et ses marques dans l'énonciation

\section{Discours rapportés : reformulation et sens}

\section{Tokiko Ishihara}

\section{OpenEdition}

\section{Journals}

Édition électronique

URL : http://journals.openedition.org/linx/959

DOI : 10.4000/linx.959

ISSN : 2118-9692

\section{Éditeur}

Presses universitaires de Paris Nanterre

\section{Édition imprimée}

Date de publication : 1 juillet 1998

Pagination : 43-52

ISSN : 0246-8743

\section{Référence électronique}

Tokiko Ishihara, «Discours rapportés : reformulation et sens », Linx [En ligne], $10 \mid 1998$, mis en ligne le 03 juillet 2012, consulté le 03 mai 2019. URL : http://journals.openedition.org/linx/959 ; DOI :

$10.4000 /$ linx.959

Ce document a été généré automatiquement le 3 mai 2019.

Département de Sciences du langage, Université Paris Ouest 


\title{
Discours rapportés : reformulation et
}

\section{sens}

\author{
Tokiko Ishihara
}

1 En s'inspirant des études théoriques sur l'hétérogénéité énonciative ${ }^{1}$, notamment celles de J. Authier autour du discours rapporté (DR), cet article s'attachera à l'étude de quelques cas de reproduction de la parole de l'autre qui se distinguent par quelques traits spécifiques : elle ne peut être représentée que sous une des modalités de DR, directe ou indirecte, mais cette transformation n'est pas réversible. Il est impossible de passer d'une forme à l'autre, sans qu'il y ait un problème d'acceptabilité, ce qui peut amener à une reformulation complète de l'énoncé. On propose de voir dans ces formes des stratégies du sujet-rapporteur; une fois que la structure du DD ou du DI a été mise en place, elle devient irréversible. Il ne s'agit pas de contester encore une fois la notion de dérivation ou de mécanismes d'intégration syntaxique. ${ }^{2}$ Notre hypothèse est que cet empêchement, loin d'être un problème de syntaxe, tient au fait que ces reproductions sont porteuses de « défauts", d'« anomalies", de «bizarreries » du dire qui gênent les opérations de transformation.

2 Si l'on considère que rapporter un discours signifie relater non pas des énoncés mais une énonciation, ces reproductions à sens unique dévoilent les traces de l'activité des sujets, plus spécifiquement celles de l'énonciation du rapporteur. Pour reprendre ce qu'a dit l'autre, l'énonciateur (E) interprète le dire du locuteur (L) avant de produire une nouvelle énonciation et par là tend à lui attribuer un sens nouveau. Ces bizarreries sont des marques de subjectivité qui constituent, comme le dit J. Authier, le champ de négociation entre l'hétérogène montré et l'hétérogène constitutif du discours ${ }^{3}$.

3 A partir de quelques exemples extraits de la presse brésilienne ${ }^{4}$ et française, notre réflexion portera sur l'étude de quelques indices, de nature diverse, que présents sur la chaîne énonciative introduisent ce type de décalage, de dislocation au niveau de l'énoncé rapporté, provoquant ainsi un déplacement du sens.

Du discours direct au discours indirect. On peut dire que la caractéristique principale du DD est son autonomie syntaxique vis-à-vis de l'énonciation citante. Le fait que le DD 
soit représenté sur le mode autonymique - énonciateur et locuteur étant séparés, le premier peut prendre une distance par rapport à l'énoncé qu'il rapporte - permet l'illusion d'une reproduction très proche des paroles, tenues pour effectivement proférées. Ainsi, le DD peut imiter une manière de dire (crier, chuchoter) ou encore garder les signes particuliers de cette profération en mimant les marques de l'oralité telles que débit de voix, prononciation, hésitation, éléments de prosodie et éléments phatiques, accent, etc ; pour y parvenir, les recours linguistiques et typographiques sont variés.

5 Ainsi, cet exemple extrait d'un article de l'hebdomadaire brésilien VEJA, qui rapporte une altercation entre un député fédéral truculent et des policiers au sujet d'infractions au code de la route.

a. - O plano de carreira de vocês eu vou acompanhar de perto do meu gabinete em Brasília. É bom vocês saírem da brigada porque vocês vão se @\#\&**! (VEJA, 17.12.97)

(Traduction : Votre plan de carrière, je vais suivre de près à Brasília. Je vous conseille de quitter la brigade parce que vous êtes foutus ${ }^{5}$ )

6 Ce qui frappe d'abord dans cet exemple c'est la représentation peu habituelle dans ce lieu, un hebdomadaire à grand tirage, d'un terme insultant, celui-ci étant remplacé par des signes typographiques. On ne peut s'empêcher de faire le rapprochement entre cette représentation graphique, pour signifier un juron ou une insulte, et une écriture typique des BDs, liée à l'image, mais plutôt rare en dehors de ce cadre. Bien sûr, les fonctions sémantique et énonciative des signes de ponctuation et des signes typographiques dans la BD sont d'un autre ordre et dépassent la simple représentation des mots interdits. Ce qui nous intéresse ici c'est leur valeur référentielle, le rapport particulier que ces signes entretiennent avec ce qu'ils désignent.

7 On peut dire que cet exemple présente deux types de difficultés distinctes mais intrinsèquement liées : la première, d'ordre pragmatique, la seconde d'ordre formel.

8 En premier lieu, on part de la constatation que le mot remplacé, en l'occurrence un verbe, existe mais est inemployable dans cet espace. Le contexte linguistique est sans ambiguïté, le terme se laisse aisément reconnaître. C'est cette relation entre le mot et la chose qui semble particulièrement intéressante, car si le mot se laisse reconnaître c'est qu'il existe bel et bien, mais l' objet qu'elle désigne est frappée d'interdit dans ce lieu. On dirait que l'indicible dans ce cas est lié à l'impossibilité de nommer, plutôt écrire, pour des raisons pragmatiques, les règles de bon usage, ce type d'insulte dans cette situation d'énonciation..

Deuxièmement, la nature idéographique des caractères typographiques semble doublement marquée. En remplaçant le mot du locuteur par les signes @\#\&** l'énonciateur signifie, d'une part, le rejet du mot de l'autre, marqué par une prise de distance à la manière des mots entre guillemets, c'est à dire, une non assomption du mot de L, même s'il l'intègre à son énonciation. Cela veut dire que la fonction des signes typographiques dans cet exemple serait bien de marquer à la fois le changement de registre de langue et les deux plans d'énonciation distincts. D'autre part, il s'agit bien là des traces que le sujet énonciateur laisse sur l'énonciation de L, une intervention qu'on reconnaît à la manière singulière de représenter le mot proféré (les points de suspension sont plus conventionnels dans ce cas-là), on dirait presque son empreinte personnelle. Cette interprétation du mot de l'autre introduit quelque part, un déplacement du sens, elle permet à $\mathrm{E}$ de réorienter l'énonciation de $\mathrm{L}$ et de montrer qu'il s'agit bien d'une autre 
énonciation. Cela renforce, par ailleurs, l'idée qu'il n'existe pas de reproduction « fidèle ", « textuelle ». En empruntant à la BD la manière de dire, E reformule et détourne à jamais le sens originel. L'énonciation de L devient ainsi ridicule, voire grotesque, outre qu'il s'agit d'une menace, d'un abus d'autorité.

Quant à l'impossibilité de transposition, nous avons dit que dans certains cas, la parole de l'autre ne peut être rapportée que sur un mode, DD ou DI, la transformation, par exemple, d'un DD en DI n'étant pas possible pour des raisons d'acceptabilité, parmi d'autres raisons. Dans l'exemple étudié, la difficulté est liée non seulement à la forme mais aussi au sens. D'une part, il y a la présence matérielle des signes typographiques @\#\&** que l'on peut accepter dans un DD mais difficilement en DI, et d'autre part, la transcription du mot puisqu'il n'est pas possible de garder les signes en DI. Il faudrait les remplacer par un équivalent plus familier, par des points de suspension ou encore par troncation à la mode de La P... respectueuse de Sartre. Reformulation ou altération, le choix n'est jamais neutre.

11 Si le DD permet de reproduire les paroles de l'autre en gardant les particularités du registre oral, ce sont ces caractéristiques mêmes qui constituent un obstacle à une transposition en DI. Dans le cas de l'exemple a, il y a d'abord une syntaxe "relâchée ", propre à l'oral (ça se dit mais ça ne s'écrit pas) avec une disposition moins rigoureuse des éléments dans la phrase, le registre de langue marqué, le point d'exclamation dont la valeur énonciative reste à interpréter entre véhemence et colère.

A moins que l'on reformule tout, on voit mal une reproduction indirecte du type

?? O deputado disse que o plano de carreira deles ele ia acompanhar de perto do seu gabinete de Brasilia e que era bom eles saírem da brigada porque eles iam se @\#\&**

Cet énoncé est difficilement acceptable, nous sommes dans le domaine du polémique, du vague, du « ça pourrait éventuellement se dire mais ça ne s'écrit pas ». Et quel traitement donner à cette manifestation matérielle du signifiant, @\#\&**, qui, en tant qu'alignement des signes non verbaux, ne peut être transposé littéralement? Le DI étant une forme homogénéisante, au contraire du DD, forme de l'hétérogène, l'énonciation citée se place sous la « dépendance » syntaxique de l'énonciation citante. Par conséquent, la démarche naturelle serait la "neutralisation », le "gommage » des marques de la subjectivité de l'énonciation de L, celle-ci étant absorbée par l'énonciation de $\mathrm{E}$.

15 Examinons ensuite deux cas de reproduction directe d'une langue étrangère, mais de nature différente :

b. La légende affirme que Charlie Chaplin, lorsqu'on lui demandait de citer la plus belle chose qu'il avait vue, répondait: "The movement of Helen Wills playing tennis. » (Le Monde, 06.01.98)

c. «Chira! Chira ! », rigolent les hommes en ondulant. (Le Monde, 11.06.98)

(Traduction: «A poil! A poil », mais peut être d'un emploi moins vulgaire, moins agressif que l'expression française)

Si dans l'exemple $\boldsymbol{a}$, c'est un élément inscrit sur le mode autonymique dans la chaîne du DD qui constitue le point d'hétérogénéité intransposable, dans $\mathbf{b}$ et $\mathbf{c}$ c'est tout l'énoncé en DD qui devient obstacle. Dans l'exemple $\mathbf{b}$, type classique concernant une langue étrangère, une reproduction en DI semble impossible sans que la forme soit malmenée par une hybridation des langues et sans que le sens devienne opaque en raison de cela :

?? Charlie Chaplin... répondait que c'était the movement of Helen Wills playing tennis.

18 Bien sûr, on peut toujours dire que le signifiant n'a qu'une importance relative et que ce qui importe c'est de signifier qu'il y a eu un acte énonciatif ; c'est une question de point 
de vue. Comme dans l'exemple précédent, c'est parce que la représentation sur le mode direct permet de distinguer les deux plans de l'énonciation qu'il est possible de garder la langue étrangère. Si l'on transpose l'énonciation de L en DI, on l'intègre à l'énonciation de $E$ et l'autonomie qui permettait justement de lire l'énoncé en langue étrangère cesse d'exister.

L'exemple c est de nature plus complexe, il ne reproduit pas une langue étrangère, mais la représentation phonique du mot en langue étrangère: chira est la représentation phonétique (tel que l'entend un Français ?) de tira, $3^{\mathrm{e}}$. personne du singulier du verbe portugais tirar (enlever, ôter) comme on le prononce au Brésil, c'est à dire, en palatalisant le phonème $/ \mathrm{t} /$, soit $/ \mathrm{tj} /$. Tchira, comme dans le mot tchache (la langue codée des jeunes des cités), serait une transcription plus proche de la prononciation française. Quelle que soit la manière dont on transcrit, cela a finalement peu d'importance, ce qui intéresse c'est qu'une telle reproduction de la parole d'autrui est une interprétation de $\mathrm{E}$ et n'est, encore une fois, possible qu'en DD: outre l'incertitude lexicale du mot en langue étrangère (c'est une représentation, en fait) et l'environnement expressif (la répétition, l'exclamation), l'absence du verbe dire (ou un autre du même type), remplacé par rigoler en ondulant augmente d'un cran la difficulté. Comment restituer cette parole collective, scandée, rythmée, presque chantée et ponctuée de rires, si ce n'est par la voie (et voix) directe?

Cette impossibilité n'est pas seulement formelle, toute reproduction de la parole de l'autre, directe ou indirecte, implique une construction nouvelle de forme et de sens, une métaénonciation que Bakhtine a bien définie : «Le discours rapporté, c'est le discours dans le discours, l'énonciation dans l'énonciation, mais c'est en même temps, un discours sur le discours, une énonciation sur l'énonciation. $»^{6}$ (p.161). Si l'on ne peut pas passer «mécaniquement » d'une forme à l'autre, la citation de Bakhtine montre bien qu'il y a une sorte de « réversibilité » linguistique : ce n'est pas seulement l'énonciation citante qui tient un discours sur l'énonciation citée, mais celle-ci agit aussi sur celle qui l'incorpore. C'est ce qui explique la présence de rigoler en ondulant dans l'exemple $\mathbf{c}$. C'est bien une manière de dire (orale et gestuelle) qu'aurait voulu restituer le rapporteur.

21 On peut montrer par d'autres exemples en DD, la capacité que ce mode a de reprendre une parole en conservant certaines marques spécifiques de la situation de communication, en raison de son statut autonymique, c'est-à-dire, en préservant la subjectivité à la fois de $\mathrm{E}$ et de $\mathrm{L}$ et pour cette raison même difficile à reproduire en $\mathrm{DI}^{7}$.

C'est le cas, par exemple, de $\mathbf{d}$, qui conserve l'expression de $\mathrm{L}$ en langue étrangère et sa forme répétée qui rappelle l'emphase donnée à l'appréciation :

d. "Very friendly, very friendly», disent les Ecossais en évoquant Paris. (Le Monde, 11.06.98)

23 Ici le problème n'est pas seulement la langue étrangère mais aussi la prosodie; pour rapporter en DI le dit des Ecossais, il faudrait traduire, éliminer la répétition, ajouter un commentaire au dire. Cet effacement des marques de l'expression orale ne va pas sans altération du contenu.

En $\mathbf{e}$ et $\mathbf{f}$, la difficulté est d'un autre ordre : c'est un registre de langue marqué, différent de la langue standard et le caractère d'expression idiomatique qui font problème.

e. «Ah les cons!", dit Iptissen, lycéenne. (Le Monde, 11.06.98)

f. « Non, mais ça va pas », dit-elle en rigolant. (Le Monde, 11.06.98) 
Dans ces formes «d'écarts de la langue » (cf. Authier), une reproduction indirecte obligerait à recourir à la traduction du discours autonyme, au sens propre du terme pour ce qui est d'une langue étrangère (d), et figuré pour e et $\mathbf{f}$. Autrement dit, ils n'ont d'intérêt qu'en tant qu'ils sont reprises, en apparence textuelles, du dit.

Dans l'exemple ci-dessous (suite de a), la présence de - rapaz ${ }^{8}$ - dont la fonction phatique ne laisse pas de doute quant à la situation de communication est une pièce importante dans la construction du sens d'autant plus qu'il est suivi de points de suspension et s'offre ainsi à l'interprétation :

g. Quando é uma autoridade, um governador ou um deputado federal, vocês têm que liberar, rapaz... (Veja, 17.12.97)

(Traduction: Quand il s'agit d'une autorité, un gouverneur ou un député fédéral, il faut libérer, jeune homme...)

Du discours indirect au discours direct. Du point de vue de la forme, si quelques reproductions de la parole de l'autre ne sont possibles qu'en DD, justement parce qu'il y a alors une autonomie totale des énonciations, le sujet énonciateur se démarquant du locuteur, il en va autrement pour le DI. Etant une forme homogénéisante, au contraire du $\mathrm{DD}$, forme de l'hétérogène, dans le DI l'énonciation citée se place sous la dépendance syntaxique de l'énonciation citante, par la médiation d'un verbe introducteur, en général un verbe de parole ou de communication, suivi d'une subordonnée introduite par la conjonction que (il dit que). L'intégration syntaxique signifie, en d'autres termes, que la parole de l'autre est entièrement prise en charge par l'énonciateur-rapporteur qui la reproduit dans son énonciation en modifiant la forme et le contenu.

La fonction du verbe introducteur de DI, parfois remplacé par des constructions verbales, semble essentiellement métaénonciative. Il introduit, au sens littéral du terme, des informations nouvelles qui reformulent le dire de l'autre en lui attribuant un autre sens. C'est sans doute en raison de cette capacité de s'approprier la parole de l'autre, de pouvoir sélectionner ce qui sera rapporté pour mieux le conditionner à la nouvelle énonciation, que le DI est considéré comme le mode qui privilégie le contenu (ou la teneur) au détriment de la forme. Il est certain que, dans certains contextes linguistiques, le choix du DI s'impose étant donné qu'il permet au rapporteur d'interpréter, d'analyser, de résumer l'énonciation de l'autre.

Par ailleurs, si le DD permet de mimer la parole proférée en reproduisant, d'une manière assez réussie, certains éléments de prosodie comme la pause, la répétition, l'hésitation, la surprise, etc, en ayant recours aux signes de ponctuation et aux signes typographiques (gras, italique, majuscules), qui exercent aussi une fonction énonciative, dans d'autres conditions linguistiques, le DI semble préférable pour restituer certaines caractéristiques de la parole, par l'intermédiaire du verbe introducteur. Il n'est pas question d'imiter la parole, mais de construire une image, une représentation du dire de l'autre.

$\boldsymbol{h}$ - Sur les collines d'Hollywood, on murmure que Marlon Brando serait prêt à témoigner en

faveur de Cristian si nécessaire. (France Soir, 21.07.90)

Dans cet exemple, la charge sémantique de murmurer, opéré par un sujet dans son essence polyphonique - on -, plus le conditionnel de serait, construisent la figure de la rumeur, autrement dit une parole qui ne peut être attribuée à un locuteur spécifique et qui ne prend sens que parce qu'elle est prise en charge par le journaliste-rapporteur.

Pour contradictoire que cela puisse paraître, que ce soit sous la forme directe ou indirecte, on serait tenté de dire qu'on peut tout rapporter, même l'indicible des gestes, 
des bruits, des silences, des attitudes. Pour ce faire, l'énonciateur interprète et évoque ces situations de communications par des gloses, des descriptions, des commentaires, ce qui peut donner des représentations du type «il a protesté en hochant la tête, par un lourd silence, en sifflant » ou alors "en guise de réponse à ma question elle a poussé un grand soupir, a éclaté de rire, a tourné le dos, a pris ma main » etc, formes abondantes dans le récit narratif. Quelquefois, seul le DD est capable de restituer une parole, une situation. C'est le cas de certains verbes français comme soupirer, s'écrier, s'émerveiller et aussi de certains verbes portugais tels que gracejar (plaisanter), cochichar (chuchoter). On peut, peut être, rapporter, ou mieux relater, mais on ne peut pas dire.

Ce que nous avons voulu rappeler brièvement dans cet article ce sont ces formes spécifiques de reprises de la parole de l'autre qui posent des problèmes, parce qu'elles comportent soit une particularité, soit une bizarrerie du dire, qui empêchent le passage d'une forme à l'autre et, par conséquent, bloquent quelque part le sens. Ces énoncés à sens unique questionnent, par ailleurs, une certaine idée de "réciprocité ", d'alternance DD-DI ou DI-DD comme allant de soi. Il ne s'agit pas seulement de syntaxe ni seulement d'acceptabilité.

Notre intention n'était pas de faire l'inventaire des formes de DD ou de DI qui présentent des difficultés, ni de les décrire, mais de souligner que le DR est une activité langagière dont la complexité ne réside pas seulement dans le choix stylistique d'une forme, mais dans le comment, dans quel but, pour qui on rapporte. Dans les cas des exemples en DD, rapporter les énoncés en DI supposerait : traduire, pour les exemples a,b,c,d ; paraphraser ou expliquer, pour e et $\mathbf{f}$; éliminer des éléments de la chaîne pour $\mathbf{g}$, et encore pour n'évoquer que les opérations les plus évidentes. Dans pratiquement tous les cas, il faudra ajouter une explication ou un commentaire. Pour l'exemple $\mathbf{h}$, en DI, on ne veut pas dire que murmurer ne peut pas rapporter un énoncé en DD. Un énoncé du type Il lui murmura au creux de l'oreille: "Je t'aime" prouve bien que cela est possible, ce qu'il semble impossible c'est de représenter visuellement murmurer, les recours typographiques étant, dans ce cas, inefficaces. .

En outre, ces cas montrent bien qu'il n'y a pas de transcription littérale du dire de quelqu'un, que ce soit en DD ou en DI, car en rapportant, le sujet énonciateur reformule et oriente le sens en signifiant qu'il s'agit bien d'une énonciation. Le DR est une activité de reformulation de forme et de sens, une opération langagière de coût linguistique très haut car cela signifie aussi mutiler, réduire, gommer, altérer.

Nous ne saurions terminer sans rappeler un dernier point, soit l'intérêt que les formes de DR, en particulier des formes «boiteuses ", c'est-à-dire qui présentent des irrégularités, ont pour la didactique et acquisition des langues. A ce titre nous rappelons la critique de Bakhtine $^{9}$ :

«La transposition mot pour mot, par des procédés purement grammaticaux, d'un schéma à un autre, sans opérer les modifications stylistiques correspondantes, n'est rien de plus qu'une méthode scolaire d'exercices grammaticaux, pédagogiquement mauvaise et inadimissible. Une telle application des schémas n'a rien à voir avec leur utilisation vivante dans la langue. » (Bakhtine, 1977, p. 176)

Apprendre à gérer la parole de l'autre est un des problèmes les plus complexes de l'enseignement/apprentissage d'une langue. S'approprier, restituer, textualiser la parole de l'autre est à la base de toute activité, orale ou écrite, en langue étrangère. Ainsi, demander, répondre, raconter, résumer, argumenter constituent les premiers gestes d'un apprenant en langue étrangère. Mais il s'agit là d'un autre sujet. 


\section{BIBLIOGRAPHIE}

ARRIVE, M., GADET, F., GALMICHE, F. (1986) La grammaire d'aujourd'hui. Paris, Flammarion.

AUTHIER-REVUZ, J. (1978) Les formes du discours rapporté. Remarques syntaxiques et sémantiques

à partir des traitements proposés. DRLAV, 17, pp. 1-87.

AUTHIER-REVUZ, J. (1992) Les non-coïncidences du dire et leur représentation méta-énonciative. Etude linguistique et discursive de la modalisation autonymique, Thèse de Doctorat d'Etat, Université de Paris VIII - Saint Denis.

AUTHIER-REVUZ, J. (1992-1993) Repères dans le champs du discours rapporté. L'information grammaticale, 55, pp. 00-00, et 56, pp. 10-15.

BAKHTINE, M. (1977) Le marxisme et la philosophie du langage, Paris, Minuit.

BENVEnISTE, E. (1966-1974) Problèmes de linguistique générale I et II, Paris, Gallimard.

combettes, B. (1990) Enoncé, énonciation et discours rapporté, Pratiques, 65, pp. 97-111.

CULIOLI, A. (1990) Pour une lingüistique de l'énonciation, Paris, Ophrys.

Ducrot, O. (1984) Le dire et le dit, Paris, Minuit.

MALDIDIER, D. (1990) (Re)lire Michel Pêcheux aujourd'hui, in L'inquiétude du discours. Paris, éd. des Cendres.

NORMAND, C., dir. (1990) La quadrature du sens, Paris, PUF.

PARRET, H., dir. (1991) Le sens et ses hétérogénéités, Paris, Seuil.

\section{Exemples}

Veja, 17.12.97

Le Monde, 06.01.98, 11.06.98.

France Soir, 21.07.90.

\section{NOTES}

1. Les références théoriques sont la théorie de l'énonciation d' E. Benveniste, le concept de dialogisme M. Bakhtine, le concept d'hétérogénéité élaborée par M. Pêcheux et A. Culioli dans la pratique développée par J. Authier.

2. L'idée que le DI est un dérivé du DD. Les opérations de transformations sont ramenées à des mécanismes de transpositions morpho-syntaxiques.

3. J. Authier. Thèse de doctorat d'état. (Voir ref. bibliographiques)

4. Veja (hebdomadaire brésilien), Le Monde, France Soir (quotidiens français)

5. En portugais les signes @\#\&** remplacent le verbe «se faire baiser».

6. M. Bakhtine, in Le marxisme et la philosophie du langage, p.161.

7. Il est possible de reproduire aussi en DI quelques traits de l'oral, à travers un verbe introducteur de parole ou de discours, mais l'enjeu est autre, car en DI il y a appropriation et intégration du discours d'autrui. 
8. Rapaz, en portugais du Brésil, signifie littéralement jeune homme. Normalement, l'emploi de rapaz, comme dans cet exemple, a une fonction phatique, mais la valeur peut changer selon les tournures. Il peut signifier irritation, comme dans ce cas, ou étonnement, il peut servir aussi à interpeller une personne du sexe masculin.

9. M. Bakhtine, in Le marxisme et la philosophie du langage.

\section{RÉSUMÉS}

Ce travail s'inscrit dans le cadre des travaux sur le discours rapporté en tant qu'activité de reformulation et construction du sens. Cette étude porte sur quelques cas spécifiques des modes de reproduction de la parole de l'autre dont la particularité est leur irréversibilité : ce sont des formes re-produites pour être comprises dans un seul sens. Le mode de discours, direct ou indirect, mis en place ne permet pas de transformations morpho-syntaxiques du type classique. Ce sont des formes que nous appellerons, provisoirement, « boîteuses » parce qu'elles présentent des «bizarreries » du dire qui contraignent finalement la parole de l'autre à un un seul mode de reproduction et contrarient une idée assez répandue de réciprocité des formes du discours rapporté. Ces écarts sont perçus comme des marques de l'activité des sujets. Une forme de l'indicible?

This paper presents a number of examples showing that some non standard utterances cannot be transposed from direct discourse to indirect discourse and reciprocally according to plain synctatical rules. The gap resulting from this transposition can be observed as eliciting a specific semantic process.

\section{AUTEUR}

\section{TOKIKO ISHIHARA}

Universidade de São Paulo (USP) 